\title{
Ectasia coronaria y miocardiopatía hipertrófica. Una entidad infrecuente.
}

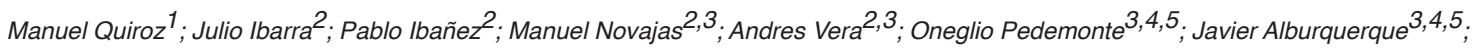 \\ Juan Eduardo Illanes ${ }^{6}$; Mauricio Aninat ${ }^{7}$, Ernesto Aránguiz-Santander ${ }^{2,3,4}$.
}

1. Cirugía cardiovascular, Hospital Regional de Antofagasta, Dr. Leonardo Guzmán, Antofagasta, Chile.

2. Cirugía cardiovascular, Hospital Dr. Gustavo Fricke, Viña del Mar, Chile.

3. Departamento de Enfermedades Cardiovasculares, Universidad de Valparaíso, Viña del Mar, Chile.

4. Unidad de cuidados intensivos cardiovasculares, Hospital Dr. Gustavo Fricke, Viña del Mar, Chile.

5. Fundación Cardiovascular Dr. Jorge Kaplan Meyer, Viña del Mar, Chile.

6. Unidad de Imagenología, Hospital Dr. Gustavo Fricke, Viña del Mar, Chile.

7. Unidad de Hemodinamia y cardiología intervencional. Hospital Dr. Gustavo Fricke, Viña del Mar, Valparaíso, Chile.

Se presenta el caso de un paciente de 54 años que consulta por angina de esfuerzo de 2 años de evolución en quien se identifica una dilatación ectásica del árbol coronario con lesiones ateroscleróticas críticas y miocardiopatía hipertrófica septal obstructiva. Una revisión bibliográfica revela que es una asociación infrecuente de la cual solo existen reportes de casos aislados.

Palabras clave: Ectasia de arteria coronaria; miocardiopatía hipertrófica; enfermedad de arterias coronarias; ateroesclerosis coronaria; angiografía coronaria.

\section{Coronary artery ectasia and hypertrophic cardiomyopathy: Case report}

We present the case of a 54-year-old patient who presented with a history of 2 years with angina. Invasive studies revealed critical coronary artery stenosis coexisting with obstructive hypertrophic miopathy.

This is a rare ascoiation with only isolated case reports.

Key words: coronary artery ectasia; hypertrophic cardiomyopathy; coronary artery disease; coronary angiography. 


\section{Introducción:}

La ectasia de arterias coronarias (EAC) es una entidad caracterizada por una dilatación de la vasculatura coronaria de forma difusa, a diferencia del aneurisma coronario que es una dilatación focal de una arteria coronaria en 1.5 veces su diámetro respecto a un segmento sano. ${ }^{1}$

La presencia de estas alteraciones se encuentra hasta en el 5\% de las coronariografías y está asociada a peor pronóstico, independiente de la presencia de enfermedad ateroesclerótica. Estas alteraciones pueden presentarse como síndrome coronario agudo o de manera asintomática, las alternativas de tratamiento son variadas y no existen directrices claras al respecto ${ }^{1,2}$.

La asociación de EAC y miocardiopatía hipertrófica $(\mathrm{MCH})$ es una entidad infrecuente, de la cual solo hay reportes de casos y existen diversas teorías sobre su etiopatogenia. ${ }^{1-3}$

\section{Presentación de caso:}

Hombre de 54 años, con antecedente de dislipidemia, hipertrigliceridemia severa y tabaquismo crónico. Presenta angina crónica estable de 2 años de evolución asociado a disnea de esfuerzos moderados y ocasional disnea paroxística nocturna.

En un test de esfuerzo con protocolo de Bruce alcanzó 4,6 METS y el test fue positivo por angor y depresión del segmento ST en región antero-lateral. Solo después de este test el paciente reveló que ocasionalmente había presentado síncope de esfuerzo. El examen físico reveló un soplo holosistólico IV/VI en foco mitral irradiado hacia la axila izquierda.

En la coronariografía se observaron lesiones en la arteria coronaria derecha con 3 dilataciones de gran volumen en tándem con estenosis pre y post aneurismáticas entre 70 a $90 \%$ del lumen. La arteria circunfleja presentaba una gran dilatación y estenosis de $70 \%$ y la arteria descendente anterior lesiones en rosario con estenosis entre 60 a $70 \%$ (Figura 1).

En el ecocardiograma 2D y Doppler color se observó un ventrículo izquierdo con hipertrofia septal acentuada de $25 \mathrm{~mm}$, insuficiencia mitral moderada secundaria a movimiento sistólico anterior del velo mitral. La fracción de eyección del ventrículo izquierdo era 73\% (Simpson) (Figura 2). Además, se efectuó un angioTAC de arterias

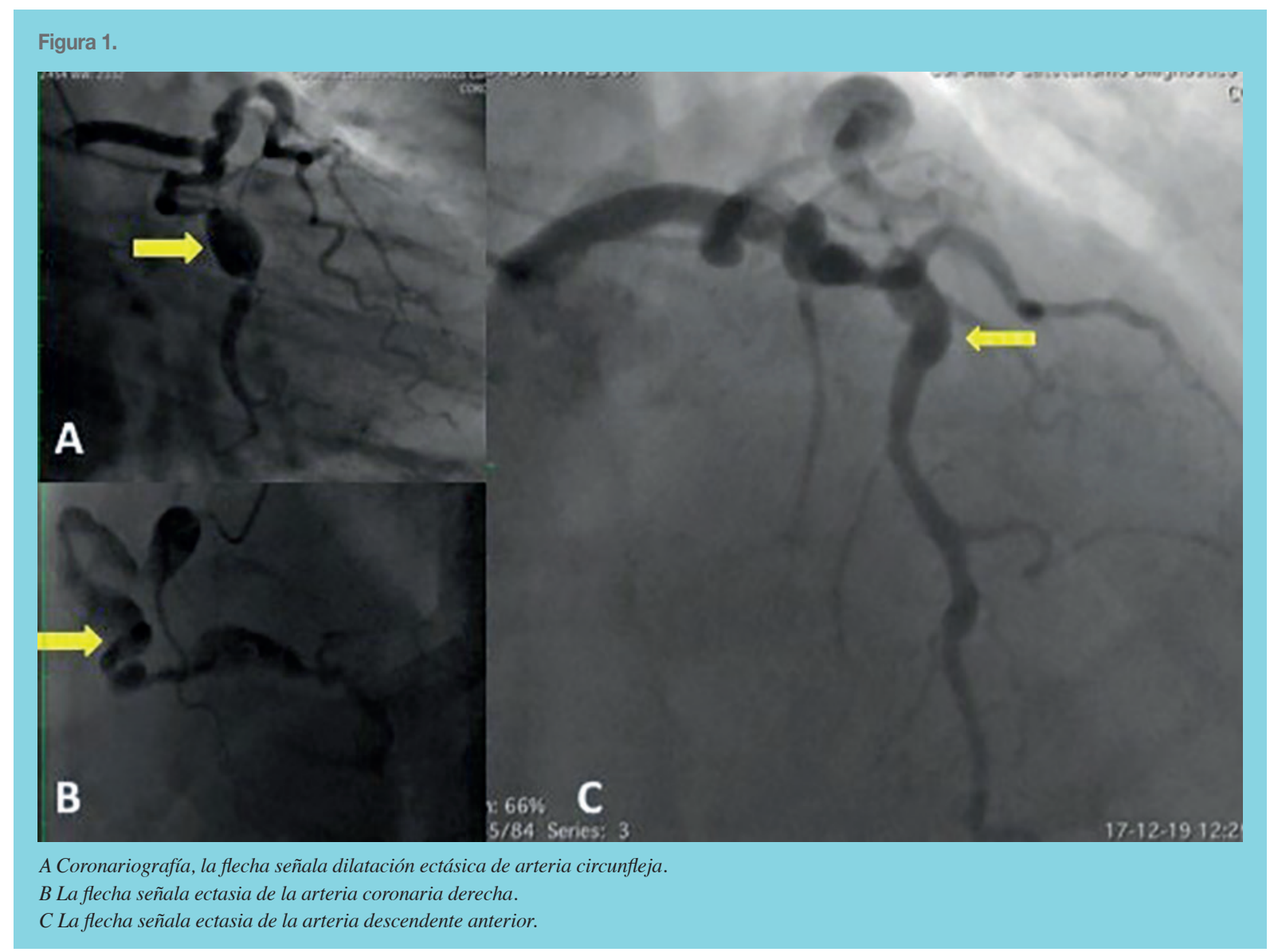


Figura 2.

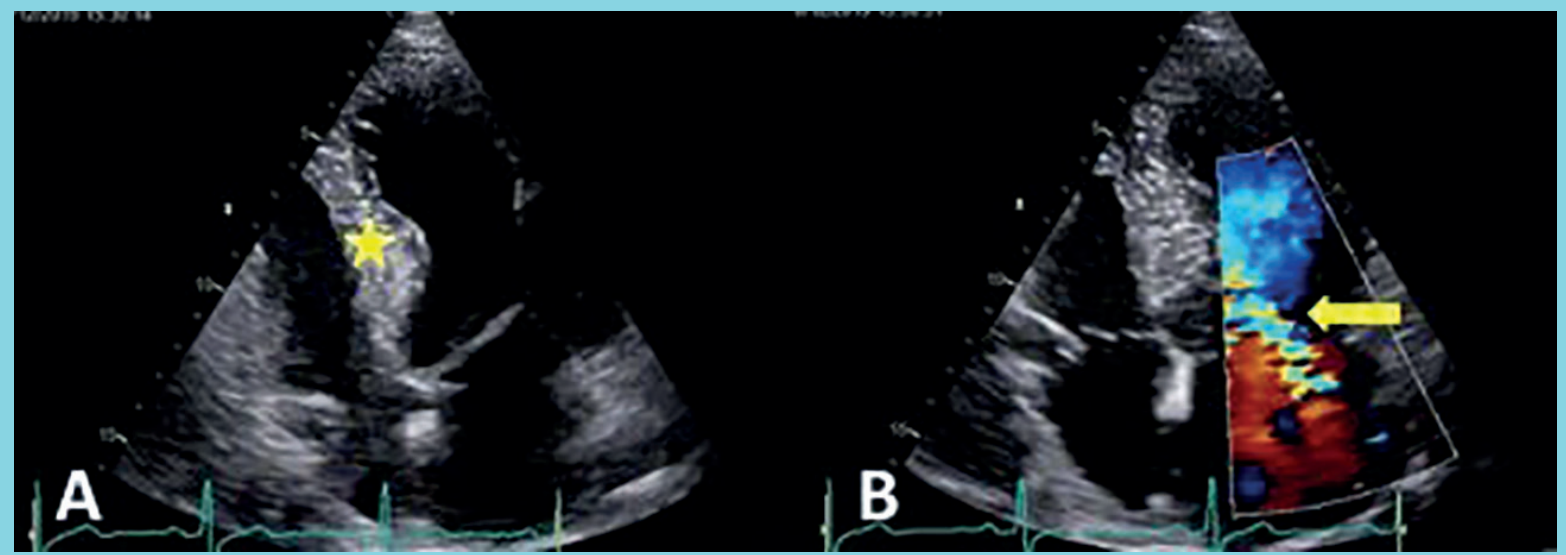

A Ecocardiografía 2D, imagen de 4 cámaras, la estrella señala hipertrofia septal.

B Ecocardiografía $2 D+$ Doppler, imagen de 4 cámaras, flecha señala insuficiencia mitral severa por efecto de movimiento anterior sistólico.

coronarias que precisó la disposición anatómica de las lesiones (Figura 3) .

El paciente fue intervenido quirúrgicamente realizándose una anastomosis de la arteria mamaria interna izquierda a la arteria coronaria descendente anterior, puente safeno aorto-coronario a la arteria descendente posterior,

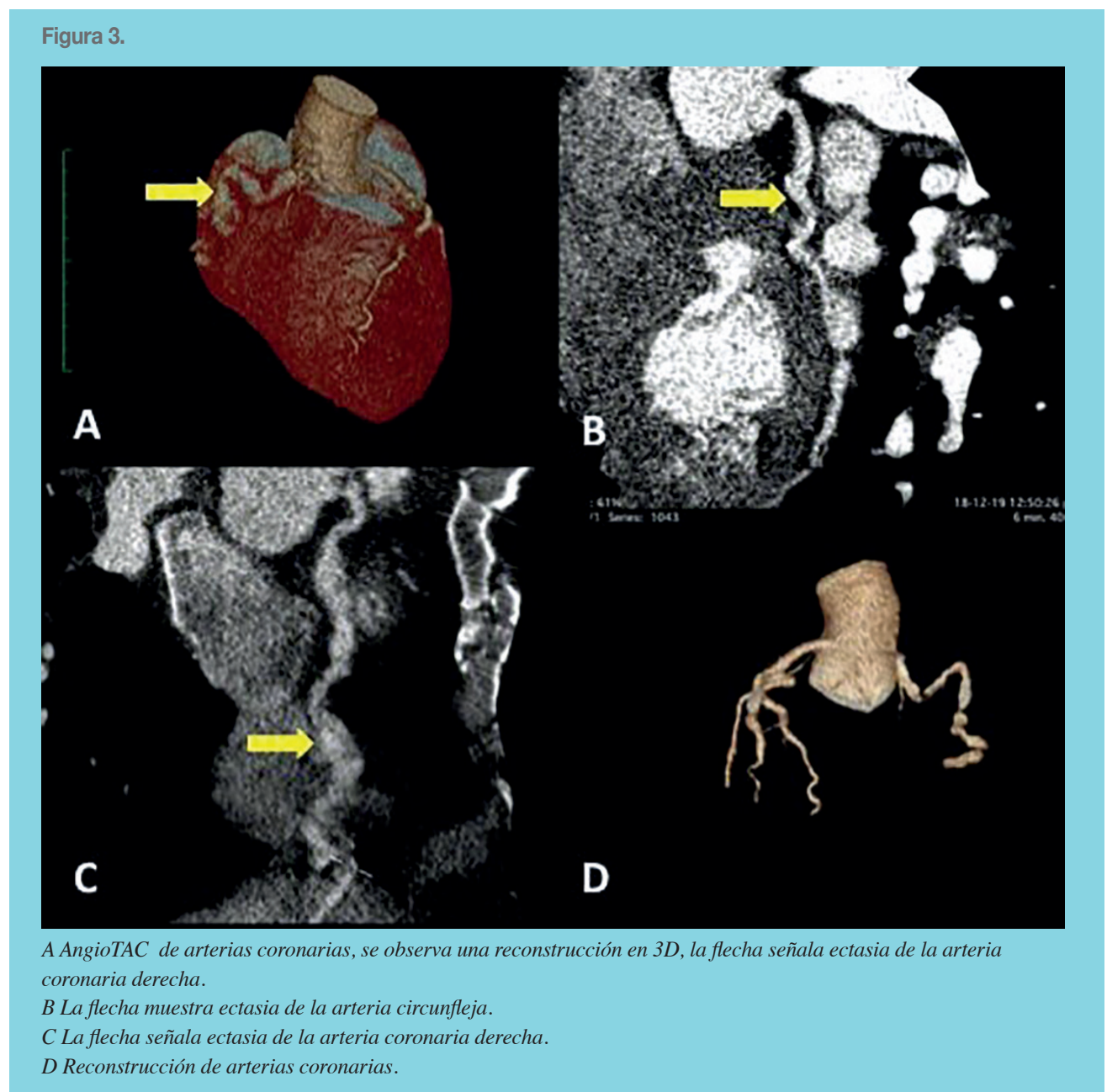




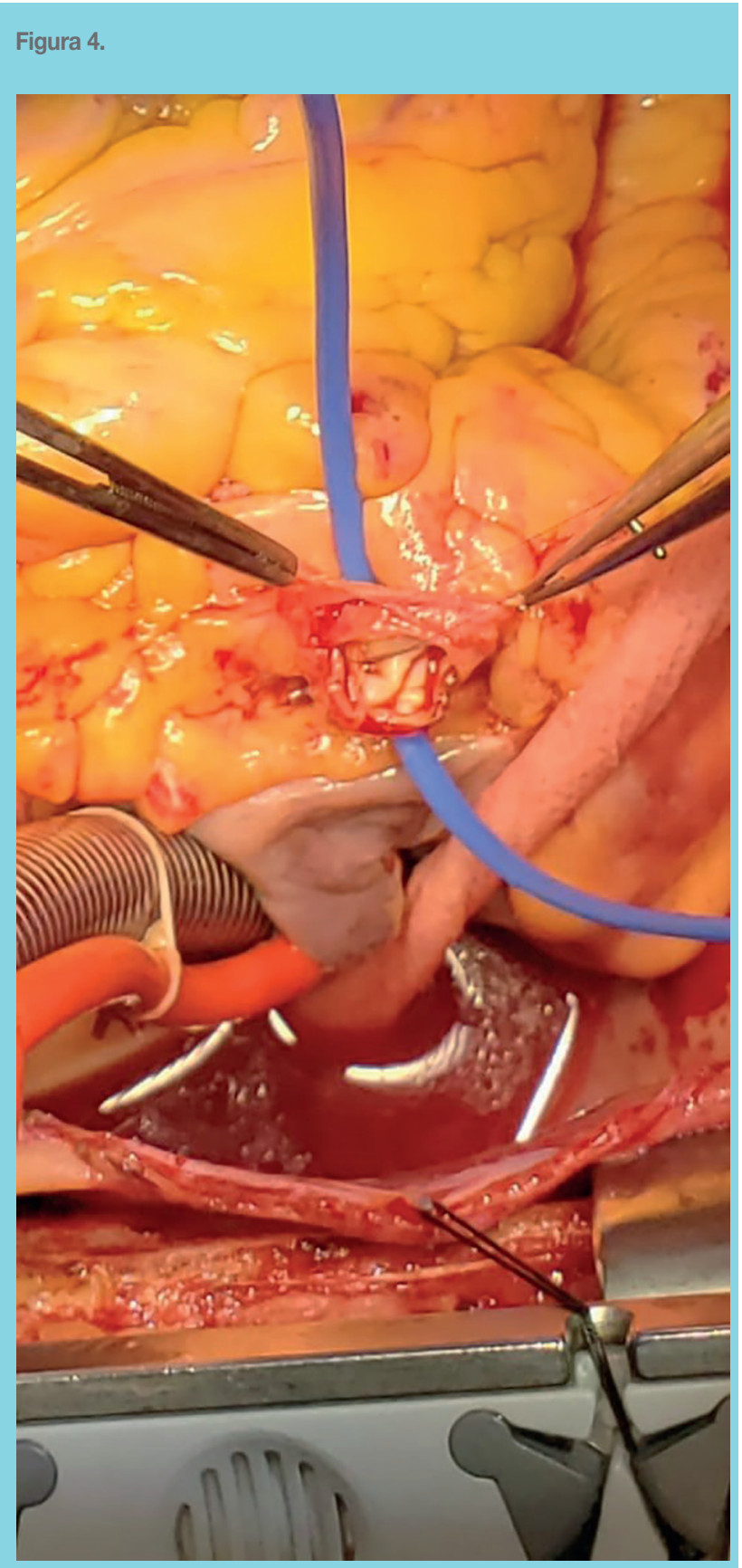

Fotografía intraoperatoria, elástico azul rodea la lesión de la arteria coronaria derecha proximal que ha sido abierto.

ligadura de aneurismas proximales y miectomía septal transvalvular (Figura 4). Los tiempos operatorios fueron 167 minutos de circulación extracorpórea y pinzamiento aórtico de 87 minutos.

Evolucionó favorablemente en la unidad de cuidados intensivos y posteriormente en sala, indicándose finalmente su alta y control ambulatorio.

\section{Discusión:}

Desde la descripción de una dilatación anormal de arterias coronarias en 1812 por Bougon, se han publicado casos clínicos aislados a nivel mundial. Hasta poco tiempo atrás se utilizó indistintamente los conceptos aneurisma de arteria y ectasia coronaria. Es importante, sin embargo, aclarar que aneurisma se refiere a una alteración focal y ectasia a una alteración difusa. 4

La etiología de la ectasia coronaria es desconocida y se sugieren diversas teorías para explicarla. Lo más frecuente es que se asocie a enfermedad ateroesclerótica que involucre más de una arteria coronaria y se explica por el remodelado de una placa que causa una dilatación en el lumen de un vaso comprometiendo la túnica media y la membrana elástica externa. También se ha reportado como una alteración congénita o asociada a diferentes cuadros infecciosos o inflamatorios como la enfermedad de Kawasaki, aneurismas micóticos sifilíticos y la periarteritis nodosa. Estas lesiones se ubican, principalmente, en los segmentos proximales y su morfología tiende a ser fusiforme con compromiso difuso del vaso, como se observó en este paciente.

La ecografía intracoronaria ha demostrado que el remodelado puede ocurrir de forma bidireccional, dependiendo de la expansión o contracción de la membrana elástica externa (MEE), sugiriendo que ocurre una remodelación expansiva exagerada de la MEE. La degradación enzimática de la matriz extracelular por metaloproteinasas y otras enzimas líticas adelgazaría la túnica media y llevaría a una inflamación crónica como componente principal del remodelado ${ }^{5,6}$.

Markis clasifica la EAC como Tipo I: Ectasia difusa de 2 o más vasos, Tipo II: Enfermedad difusa en un vaso y enfermedad localizada en otro, Tipo III: Enfermedad difusa en un vaso y Tipo IV: enfermedad localizada o segmentaria. El presente paciente se clasificaría en Tipo I con afectación de todos los vasos. ${ }^{7}$

No existe evidencia respecto a las alternativas de tratamiento, debido a lo infrecuente de esta asociación. Sin embargo, para cada entidad por separado se puede definir el manejo y así la ectasia coronaria asociada a lesiones coronarias críticas puede resolverse mediante angioplastía y stent medicado, dependiendo de la ubicación y la magnitud de la estenosis. Si no existe asociación a lesiones críticas coronarias se desconoce la historia natural con claridad, pero se sabe que se asocia a cardiopatía coronaria a futuro y la prevención secundaria mediante anti-agregación plaquetaria podría tener 
un rol ${ }^{1,2}$. Cuando existen lesiones coronarias complejas asociadas a dilataciones saculares el manejo es quirúrgico incluyendo apertura de la lesión sacular, ligadura de vaso proximal y distal y puente aorto-coronario, como se realizó en este caso.

Respecto a la miocardiopatía hipertrófica asimétrica con compromiso septal su manejo corresponderá a la gravedad de este y puede ser de tipo médico con betabloqueo y observación con ecocardiografía para determinar progresión. Si requiere intervención puede realizarse alcoholización de una rama septal mediante técnicas de cardiología intervencional o puede ser candidato a cirugía de miectomía septal transvalvular como fue en este paciente ${ }^{1-3}$.

La alteración del miocardio en forma de hipertrofia esta reportada en series de casos y se postula como una posible causa o factor desencadenante de ectasia coronaria. Las zonas ectásicas de las arterias coronarias corresponderían a zonas sometidas a tracción debido a la hiper- trofia miocárdica que actuaría como puente muscular y causaría cese del flujo coronario durante la sístole, lo que ha sido evidenciado en reportes de casos ${ }^{1-3}$. La elevada presión intraluminal y el consiguiente estrés por tensión, especialmente, durante la sístole ventricular podría promover la transformación ectásica de las arterias coronarias ${ }^{2}$.

En nuestro caso, es difícil determinar la fisiopatología probable de los hallazgos. A su vez, se dificulta llegar a la etiología del síntoma principal, angina, que puede deberse a isquemia miocárdica o a obstrucción del tracto de salida del ventrículo izquierdo. Debido al conocimiento de las alteraciones genéticas en la miocardiopatía hipertrófica, consideramos que no se debe pensar en la asociación de EAC con miopatía hipertrófica como dos entidades aisladas, sino más bien como una asociación de probable causa y efecto con factores genéticos y multifactoriales compartidos.

\section{Referencias:}

1- KAWSARA A, NÚÑEZ GIL IJ, ALQAHTANI F, MORELAND J, RIHAL C S, ALKHOULI M. Management of Coronary Artery Aneurysms. J Am Coll Cardiol Intv. 2018;11 (13): 1211-23.

2- ZOGRAFOS T, KOKLADI M, KATRITSIS D. Coronary artery ectasia and systolic flow cessation in a patient with hypertrophic cardiomyopathy: A case report. International Journal of Cardiology. 2010; 145: e114-e5.

3- SAOTOME M, SATOH H, UEHARA A, KATOH H, TERADA H, HAYASHI H. Coronary ectasia with slow flow related to apical hypertrophic cardiomyopathy--a case report. Angiology. 2005; 56(1):103-6.

4- JARCHO S. Bougon on coronary aneurysm. Am J Cardiol. $1969 ; 24: 551-3$.
5- ANTONIADIS AP, CHATZIZISIS YS, GIANNOGLOU GD. Pathogenetic mechanisms of coronary ectasia. Int J Cardiol. 2008; 130: 335-43.

6- HASHEMI MJ, PETROSSIANS AA. Diffuse arterial ectasia with hypertrophic cardiomyopathy. Jpn Heart J. 1993; 34: 235-8.

7- MARKIS JE, JOFFE CD, COHN PF, FEEN DJ, HERMAN MV, GORLIN R. Clinical significance of coronary arterial ectasia. Am J Cardiol. 1976; 37: 217-22.

8- GIELEN S, STRASSER RH, KUBLER W, HALLER C. Images in cardiovascular medicine. Coronary artery ectasia and systolic flow cessation in hypertrophic cardiomyopathy. Circulation. 1998; 97: 2372-4. 